\title{
ArcheoSciences
}

Revue d'archéométrie

\section{Étude tracéologique de la perforation. Application aux perles gravettiennes de l'abri Pataud, du Blot, des Peyrugues}

Use Wear and Perforation Study. Application to Gravettian Beads from Abri

Pataud, le Blot and les Peyrugues

\section{Jean Rodière}

\section{(2) OpenEdition \\ Journals}

\author{
Édition électronique \\ URL : https://journals.openedition.org/archeosciences/3241 \\ DOI : 10.4000/archeosciences.3241 \\ ISBN : 978-2-7535-1849-0 \\ ISSN : $2104-3728$ \\ Éditeur \\ Presses universitaires de Rennes \\ Édition imprimée \\ Date de publication : 30 avril 2011 \\ Pagination : 273-281 \\ ISBN : 978-2-7535-1847-6 \\ ISSN : $1960-1360$ \\ Référence électronique \\ Jean Rodière, « Étude tracéologique de la perforation. Application aux perles gravettiennes de l'abri \\ Pataud, du Blot, des Peyrugues », ArcheoSciences [En ligne], 35 | 2011, mis en ligne le 30 avril 2013, \\ consulté le 02 mars 2022. URL : http://journals.openedition.org/archeosciences/3241 ; DOI : https:// \\ doi.org/10.4000/archeosciences.3241
}




\title{
Étude tracéologique de la perforation. Application aux perles gravettiennes de l'abri Pataud, du Blot, des Peyrugues
}

\author{
Use Wear and Perforation Study. Application to Gravettian Beads \\ from Abri Pataud, le Blot and les Peyrugues
}

Jean RoDiÈRE*

\begin{abstract}
Résumé : Cet article a pour but l'étude des modes de perforation des perles préhistoriques par la détermination de différents paramètres, la vérification de la validité de ces différents paramètres par l'expérimentation, les analyses et comparaisons entre les perles expérimentales et les perles préhistoriques par les examens non destructifs au Microscope Electronique à Balayage (MEB) et au micro-rugosimètre.
\end{abstract}

\begin{abstract}
The objective of this article is the perforation process of the prehistorically beads by the determination of the different parameters, the verification of the validity of different parameters by experimentation, the analyses and comparisons between the experimental beads and prehistorically beads by the investigation of nondestructive technique with Scanning Electronic Microscope (SEM) and microtopography.
\end{abstract}

Mots clés : perforation, expérimentation, tracéologie, perle gravettienne, analyse non destructive, MEB, microrugosimètre.

Keywords : perforation, experimental approach, use wear, gravettian bead, non destructive analysis, SEM, microtopography.

\section{INTRODUCTION}

La perforation est une étape délicate dans le façonnage des objets préhistoriques pour tous les types de matériaux : lithique, os, ivoire, bois animal ou même bois végétal généralement disparu. Cette étape est d'autant plus délicate quand il s'agit de perforer des objets de petites dimensions tels que des perles.

Ces perforations, dans le cas des perles de l'abri Pataud du Blot et des Peyrugues (fig. 1), ont un but utilitaire pour assurer la suspension de certaines parures, ou pour fixer un objet sur un vêtement. Cependant les perforations pouvaient servir également d'éléments décoratifs tel que celles qui entourent cette rondelle du Mas d'Azil, ou bien ce décor en cupules ornant cette pendeloque de Sungir (fig. 2-3).

On peut également observer cette recherche décorative à des périodes plus récentes. Par exemple sur cette palette à fard égyptienne du $\mathrm{III}^{e}$ millénaire, la perforation de l'œil de l'oiseau est inscrite dans un triangle et n'est pas circulaire (fig. 4). Travail minutieux réalisé à l'archet avec trois incli-

* Laboratoire du Centre de Recherche et de Restauration des Musées de France, UMR 171, CNRS Palais du Louvre - Porte des Lions. 14 quai F.-Mitterand. 75001 Paris. 


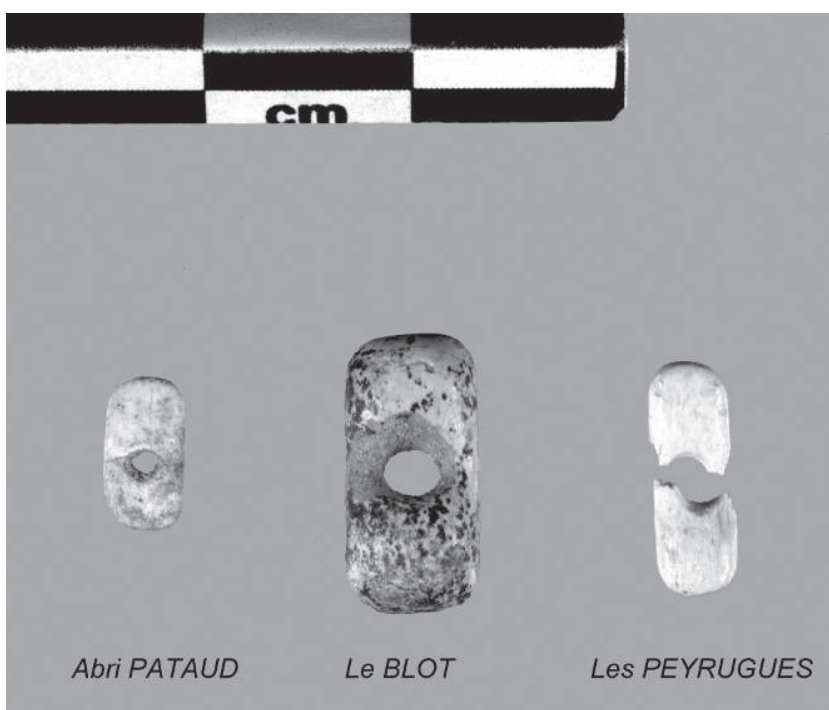

Figure 1 : Perforation pour suspension, cas de perles gravetiennes. Figure 1: Perforation for suspension, case of ravettian beads.
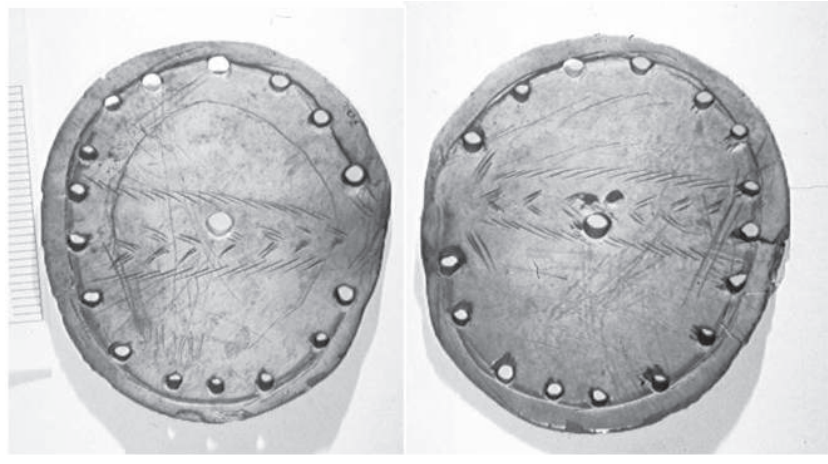

Figure 2 : (Voir planche couleur) Rondelle du Mas d'Azil. Figure 2: (See colour plate) Disk of Mas d'Azil.

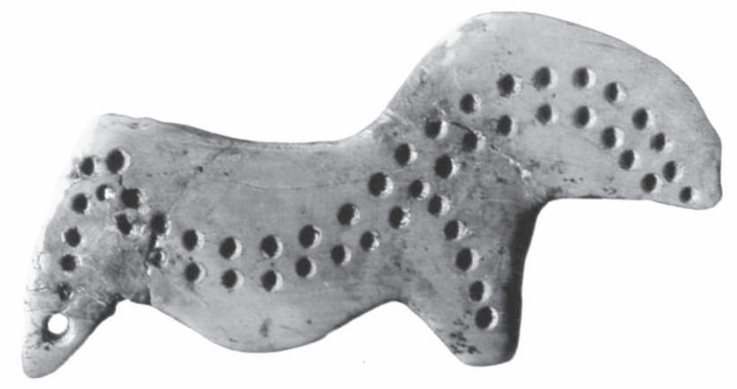

Figure 3 : (Voir planche couleur) Pendeloque de Sungir. Figure 3: (See colour plate) Pendant of Sungir.
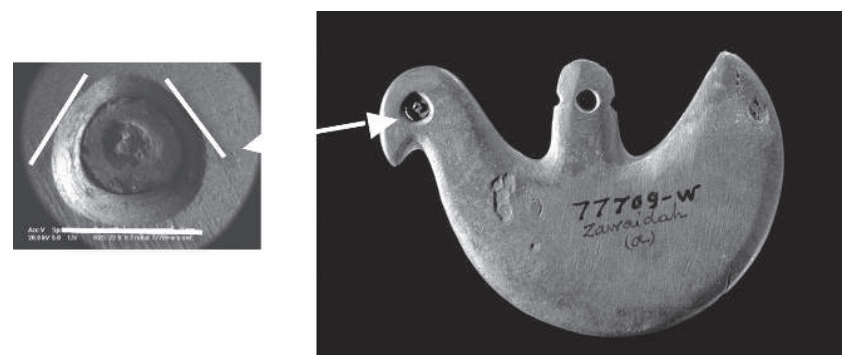

Figure 4 : Palette égyptienne du III millénaire. Figure 4: Egyptian plaque of III'd millennium.

naisons différentes de l'axe du perçoir. Cette méthode de perforation à l'archet permet une rotation multi-tours. Elle est apparue au cours du mésolithique.

Cette étude a pour but de rechercher le mode opératoire de la perforation des perles du paléolithique. Nous analyserons les paramètres liés à la perforation, suivis d'une simulation géométrique montrant l'influence de certains de ces paramètres sur la forme de la perforation, ce qui nous emmène à un exemple d'expérimentation de perle.

Enfin, nous mentionnerons le matériel d'analyse, spécialement le microrugosimètre, permettant d'observer les caractéristiques des perforations de pièces archéologiques.

\section{DÉTERMINATION DES PARAMÈTRES LIÉS À LA PERFORATION}

On peut distinguer deux types de paramètres : ceux liés à l'outil qu'est le perçoir ou micro-perçoir, l'autre dépendant de la manière dont l'artisan l'utilise.

\section{Le perçoir}

Le perçoir utilisé au Paléolithique est un outil en matière dure, souvent en silex, de forme pyramidale. Les 2 arrêtes latérales sont tranchantes et forment entre elles un angle appelé angle d'ouverture (fig. 5).

Les arrêtes tranchantes de cet outil peuvent présenter des discontinuités angulaires. On appellera angle d'épaulement ce changement d'orientation. Cet angle peut être positif ou négatif. (fig. 5).

\section{La tenue du perçoir par l'artisan}

L'axe du perçoir n'est pas systématiquement perpendiculaire à la surface à perforer, cet angle sera appelé angle d'incidence. 


\section{l'outil : - angle d'oun erture \\ - angle d'éponilement \\ la tenue de l'outil: -rangle d'incidlence \\ - angle de rotation \\ - positionnement des aces ( cas de perforation biconique)}

Figure 5 : Paramètres liés à la perforation.

Figure 5: Parameters bound with the perforation.

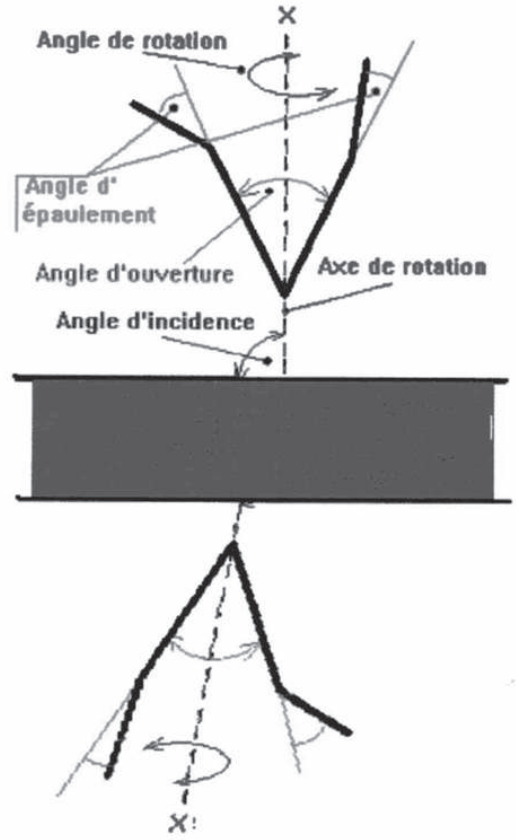

Au Paléolithique supérieur la perforation se faisait par un mouvement alternatif de semi-rotation d'une amplitude inférieure ou égale à $180^{\circ}$. Au Mésolithique l'utilisation de l'archet a permis de réaliser des perforations multi-tours. D. Stordeur et G. Pion le mentionnent lors d'une analyse d'une plaquette perforée de La Fru (Stordeur et Pion, 1993).

La force d'appui ne sera pas prise en considération; ce paramètre ne joue essentiellement que sur la durée de réalisation du percement.

L'oscillation de l'axe de l'outil peut être considérée comme négligeable. L'expérience montre que ce paramètre, important en début de perforation, est minoré en cours de pénétration, le perçoir étant guidé par les flans du volume perforé.

\section{Cas de la bi-perforation}

La perforation est généralement biconique. Le paramètre lié au positionnement relatif des axes en angle d'incidence et en écartement linéaire pour chacun des deux perçoirs vient s'ajouter aux paramètres précédents (fig. 5).

\section{Analogie géométrique}

Les simulations en géométrie descriptive permettent de mettre en évidence l'importance de ces paramètres. Deux exemples vont le montrer (fig. 6-7).

- Influence de l'angle d'incidence Dans cet exemple (fig. 6) l'axe du perçoir est décalé tous les $5^{\circ}$ entre $0^{\circ}$ et $20^{\circ}$ par rapport à l'axe perpendiculaire à la surface. La bi-perforation simulée est réalisée avec deux perçoirs identiques de forme simple c'est-à-dire de section triangulaire, d'angle d'ouverture de $60^{\circ}$ et animés d'un angle de semi-rotation de $+/-180^{\circ}$. La variation de l'angle d'incidence s'effectue uniquement sur le perçoir de la face supérieure. La partie encadrée montre la déformation de la perforation, c'est une vue de dessus de la surface perforée. L'ovalisation de la partie hachurée est uniquement due au perçoir supérieur. On observe également le décentrement de la zone blanche qui est la jonction des deux perçoirs. C'est la perforation ellemême.

- Influence de l'angle de rotation La simulation présentée est faite dans le cas de la bi-perforation (fig. 7). Le perçoir de la face supérieure possède un angle d'épaulement. L'axe de rotation du perçoir est perpendiculaire à la surface à perforer. Le paramètre variable est l'angle de semi-rotation pour lequel trois valeurs ont été choisies : $+/-360^{\circ},+/-180^{\circ}$ et $+/-120^{\circ}$. Cette dernière valeur est expérimentalement la plus naturelle. On observe dans ce cas un pincement de la figure obtenue. L'expérimentation sur une plaque de talc montre l'identité de forme (fig. 8).

De nombreuses pièces archéologiques présentent ce type de déformation. C'est le cas de cette rondelle perforée de Bedeilhac (fig. 9) ou des perles d'origine diverses par exemple la perle 30 de l'abri Pataud (fig. 9).

Il est à noter que cette valeur de $120^{\circ}$ est un ordre de grandeur que l'on retrouve sur les perforations utilisant la technique de semi-rotation. 
Paramètre : angle d'incidence

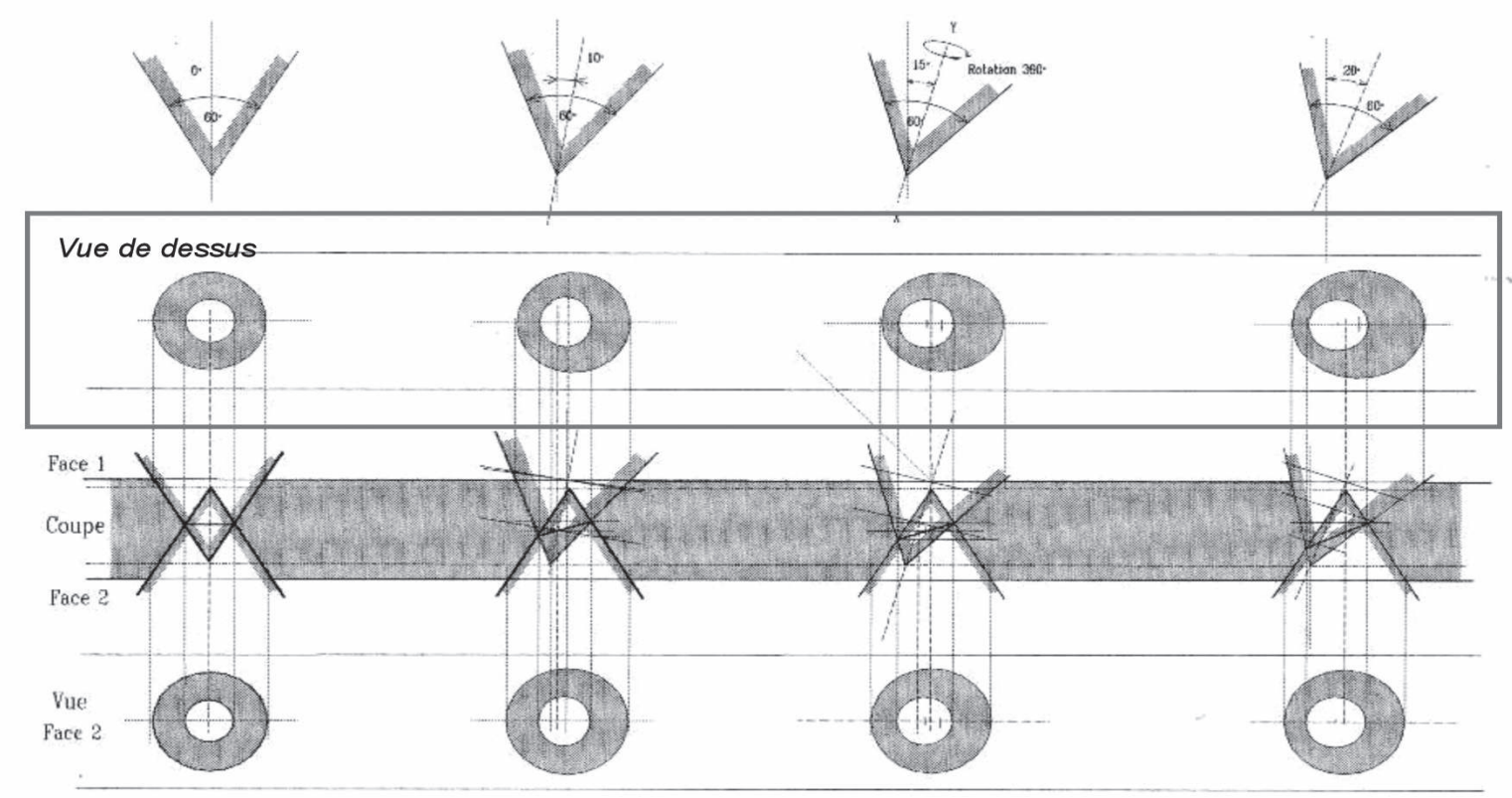

Figure 6 : Influence de l'angle d'incidence du perçoir.

Figure 6: Influence of the incidence angle of the flint tool.

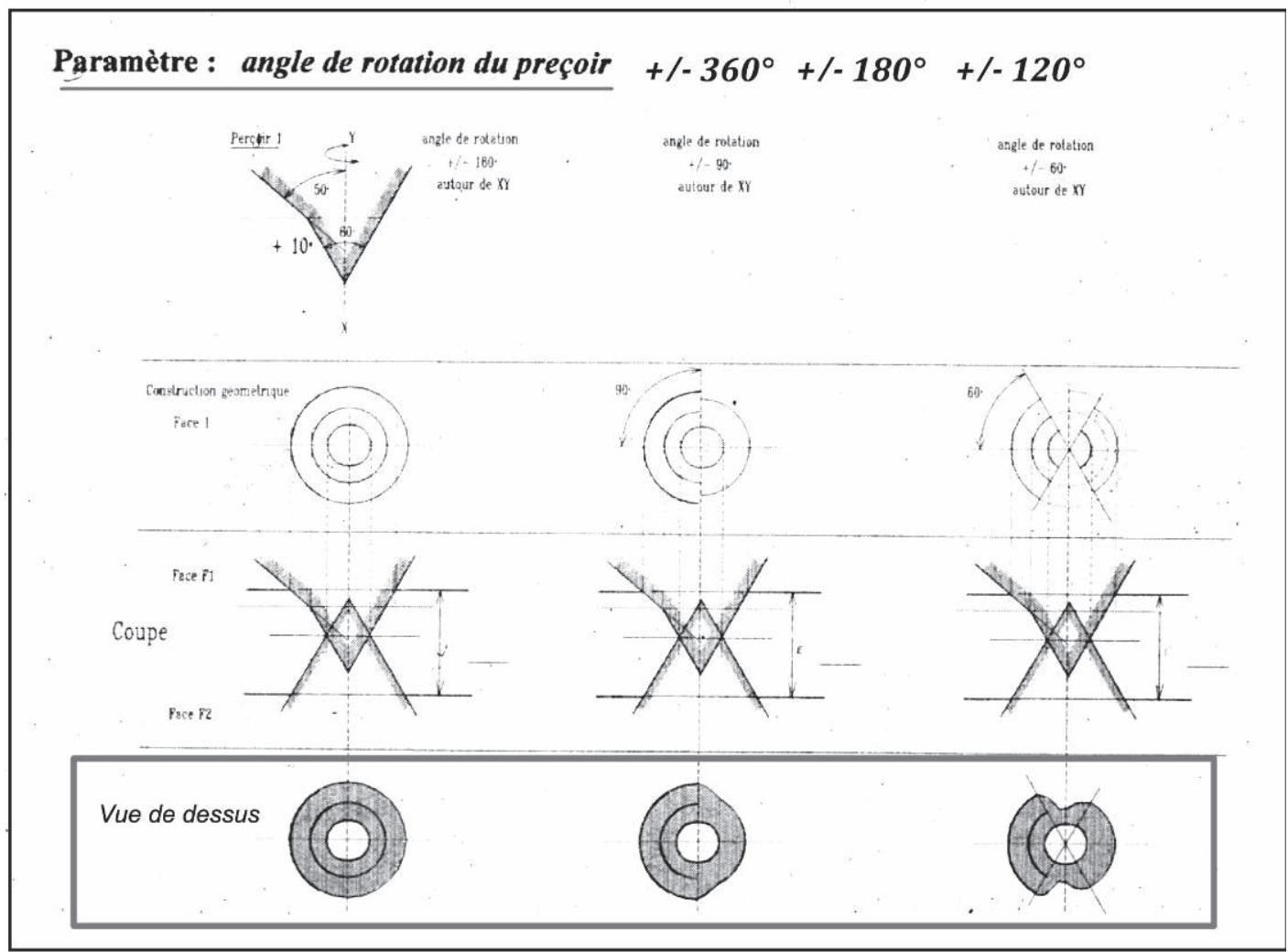

Figure 7 : Influence de l'angle de semirotation du perçoir. Figure 7: Influence of the semi-rotation angle of the flint tool. 


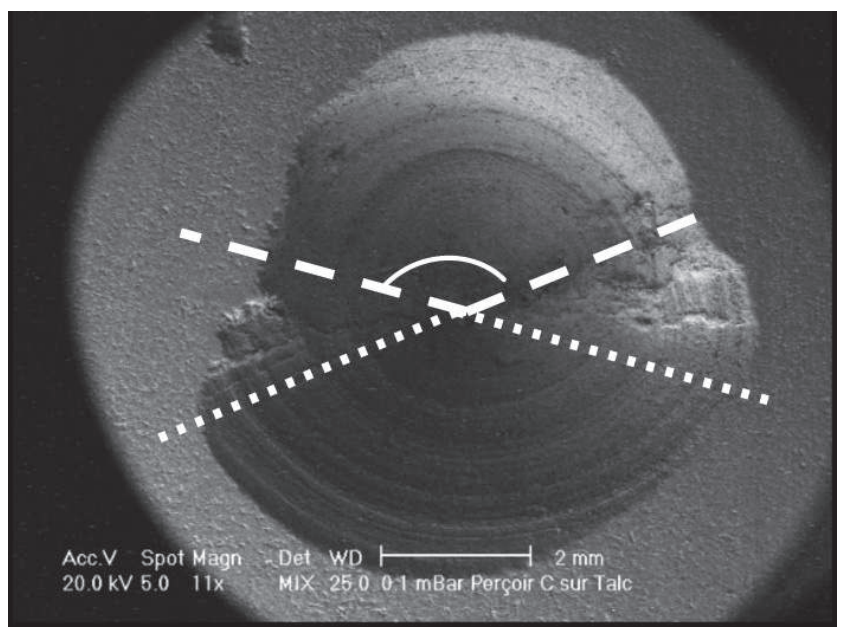

Figure 8 : Expérimentation sur plaque de talc. Cas de semi-rotation de $120^{\circ}$, le perçoir a un angle d'épaulement.

Figure 8: Experimental study on talc plate. ase of the semi-rotation of $120^{\circ}$, the flint tool has a rupture angle.
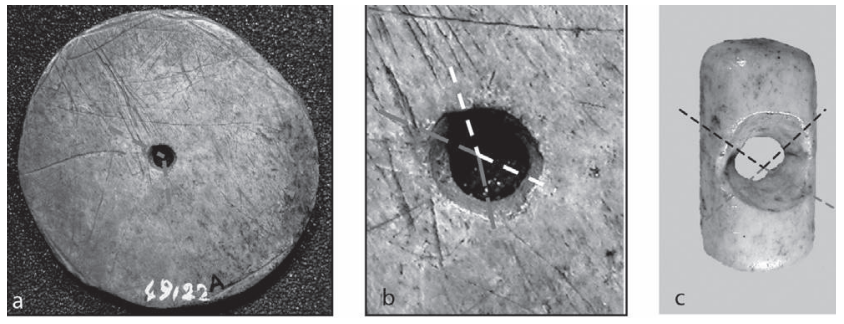

Figure 9 : a-b/ Exemple de semi rotation Rondelle perforée de Bédeilhac. c/ perle 30 de l'Abri Pataud.

Figure 9: Example of semi-rotation angle in the perforation. al the Bedeilhac disk; b/ detail; cl bead $n^{\circ} 30$ of Abri Pataud.

\section{EXPÉRIMENTATION À PARTIR DE PERLE EN STÉATITE}

La perforation est une étape délicate. Elle est réalisée avant la mise aux dimensions finales et le polissage. C'est tout au moins le cas de perles ou de pendeloques de petites dimensions, la minceur de la paroi en est la raison. L'expérimentation suivante va l'illustrer.

Elle est faite dans le cadre d'une étude sur le mode opératoire de fabrication de perles magdaléniennes en stéatite des Peyrugues de la couche 3 (fig. 10a). Ses dimensions sont : $6 \mathrm{~mm}$ de longueur sur $3 \mathrm{~mm}$ dans sa plus grande largeur et $-2 \mathrm{~mm}$ d'épaisseur avec une perforation $\leq 1 \mathrm{~mm}$. La stéatite, minéral proche du talc, a une dureté de 1 dans l'échelle de Mohs, elle est donc très tendre. Des tests sur talc ont montré que différents types de perçoirs pouvaient être utilisés
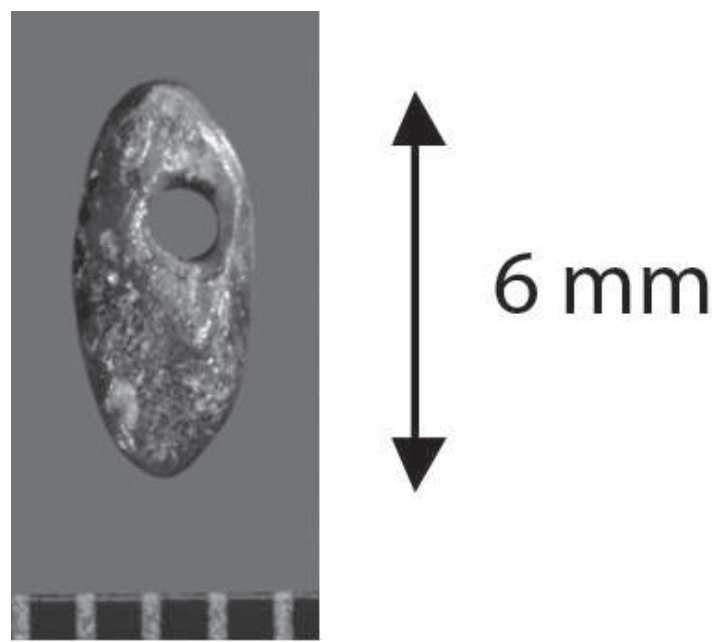

Figure 10a : Perle en stéatite des Peyrugues.

Figure 10a: Steatite bead of les Peyrugues.
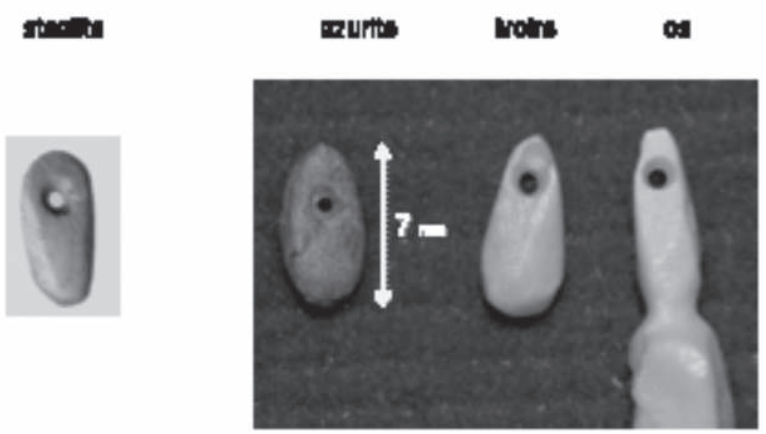

Figure10b : Perles expérimentales réalisées avec des matériaux différents.

Figure10b: Experimental beads made with different materials.

avec succès : silex, os, ivoire, et même bois en genévrier. Les comparaisons de tracéologie entre les perles archéologiques et les perles expérimentales ont montré que les perforations avaient été réalisées avec des outils en silex. L'irrégularité de la paroi active de l'outil est due aux éclats de la taille de façonnage du perçoir et se retrouvent sur les flans de la perforation (Rodiere 1996).

Cette étude a été élargie à d'autres matériaux lithiques de dureté plus grande : calcaire, bauxite (dureté 3-4), azurite (dureté 4-5) ainsi qu'ivoire et os (fig. 10b). Seul le perçoir en silex est utilisable. Le tableau 1 montre le temps mis pour perforer les perles de ce type en fonction des différents matériaux.

La tenue de la perle entre le pouce et l'index permet le façonnage aussi bien de la perforation que des finitions par abrasion et par polissage malgré leurs petites dimensions (6 $\mathrm{mm} \times 3 \mathrm{~mm} \times 1,5 \mathrm{~mm})$. 


\begin{tabular}{|c|c|c|c|}
\hline $\begin{array}{c}\text { Matériau des } \\
\text { perles }\end{array}$ & $\begin{array}{c}\text { Temps de } \\
\text { perforation }\end{array}$ & $\begin{array}{c}\text { Temps total de } \\
\text { façonnage }\end{array}$ & $\begin{array}{c}\text { Dureté } \\
\text { échelle MOHS }\end{array}$ \\
\hline Stéatite & 2 minutes & 15 minutes & 1 \\
\hline Talc & 2 minutes & 15 minutes & 1 \\
\hline Calcaire & 6 minutes & 30 minutes & 2 à 3 \\
\hline Bauxite & 15 minutes & 60 min & 4 à 5 \\
\hline Azurite & 18 minutes & 70 min & 4 à 5,4 \\
\hline Genévrier & 7 minutes & 30 minutes & \\
\hline Buis & 10 minutes & 60 min & \\
\hline Os & 12 minutes & 60 min & \\
\hline $\begin{array}{c}\text { Ivoire } \\
\text { d'éléphant }\end{array}$ & 15 minutes & 60 min & \\
\hline $\begin{array}{c}\text { Ivoire de } \\
\text { Mammouth }\end{array}$ & 12 minutes & 60 min & \\
\hline
\end{tabular}

Tableau 1 : Temps de perforation et de façonnage de perle en fonction des matériaux utilisés.

Table 1: Timing of perforation and shaping of bead for different materials.
Cette opération de perforation était précédée par un marquage initial de positionnement soit par piquetage pour les matériaux lithiques, soit par grattage comme on peut l'observer sur certaines dents perforées.

Lexpérimentation sur ce type de perles des Peyrugues s'est poursuivie par un test d'usure sur des perles montées en collier (fig. 11). Les perles en stéatite étaient disposées 2 par 2, chaque groupe étant séparé par des dentales, (coquillages marins provenant de la Méditerranée ou de l'Atlantique [Taborin, 2004]) trouvés sur le site à proximité des perles en stéatite.

Le collier est placé contre un support en peau non tannée, incliné à $15^{\circ}$, sur une table vibrante avec des fréquences variables donnant ainsi des mouvements aléatoires à l'ensemble du collier. L'impact dentale/perle est net et fait apparaître un évasement au niveau de la perforation, évasement que l'on retrouve sur la perle archéologique (fig. 12).

\section{a/}

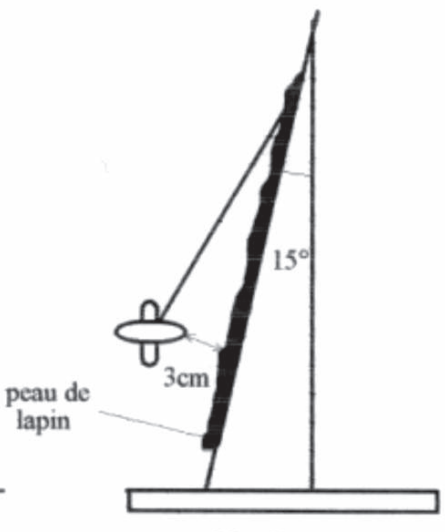

table vibrante
Figure 11 : Test d'usure sur table mental de perles séparées par des dentales b/.

Figure 11: Test on vibrating table al of an experimental of beads separated by dentalium shells $b /$. vibrante a/ d'un collier expéri-
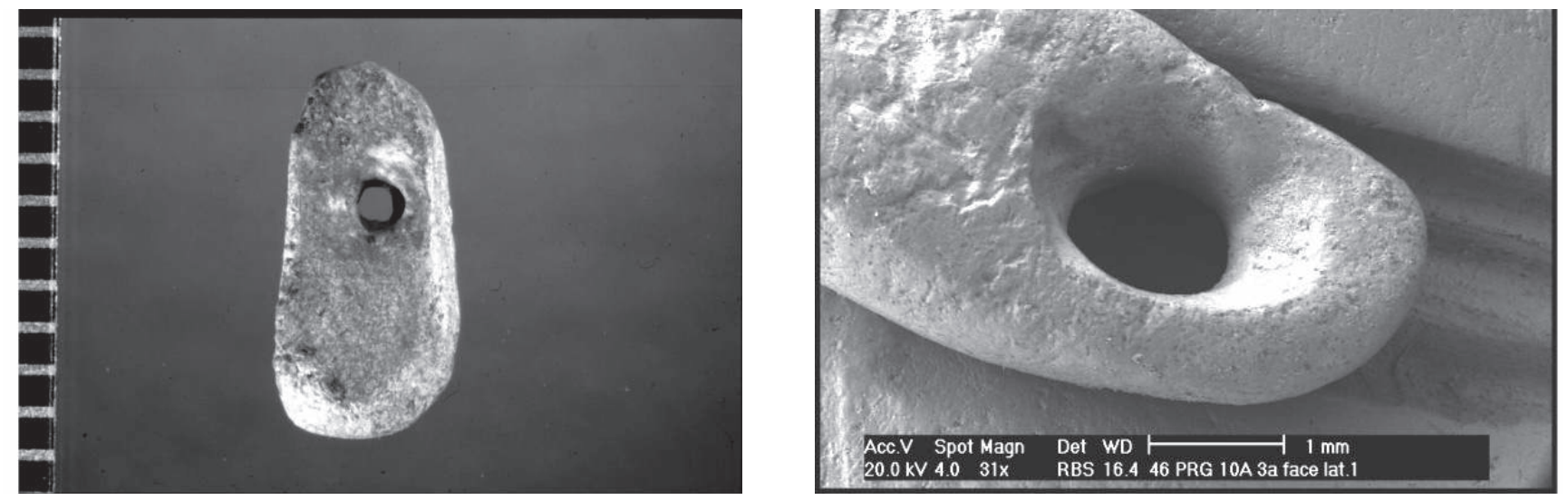

Figure 12 : a) Usure par contact entre dentale et perle provenant du collier expérimental; b) Comparaison avec une perle archéologique des Peyrugues présentant un évasement au niveau de la perforation.

Figure 12: a) contact use by abrasion between dentallium shell and bead of the experimental necklet; b) comparaison with archeological bead of Peyrugues. 


\section{Méthodes D’analyse}

Pour l'étude des perforations nous avons utilisé une binoculaire, le Microscope Electronique à Balayage (MEB) ainsi que le Microrugosimètre. Ce sont des équipements très complémentaires. Le MEB permet de réaliser des images à fort grossissement avec une grande profondeur de champ, de définir la nature des différents éléments constituant la surface observée et même d'en établir une cartographie. Le microrugosimètre permet de quantifier les irrégularités de surface de l'objet : stries, courbure de la surface, rupture de planéité...

Nous décrirons uniquement les possibilités de ce dernier équipement, le MEB étant devenu un équipement d'analyse fréquemment employé.

Le microrugosimètre est un instrument d'analyse des surfaces par balayage optique sans contact mécanique, donc sans risque de perturbation de l'objet. C'est un équipement de micromesure STIL avec capteur optique haute résolution CHR 150. Il a été utilisé notamment pour l'analyse de plaquettes de La Marche (Mélard, 2008).
Son principe est de focaliser une source de lumière blanche sur la surface à observer par l'intermédiaire d'une optique à chromatisme axial. La lumière est diffractée uniquement dans l'axe de l'optique et une seule longueur d'onde lumineuse est focalisée sur l'objet (fig. 13-14). Un retour optique renvoie le signal lumineux sur un spectromètre. L'analyse se fait par balayage de la surface à observer.

Plusieurs optiques sont disponibles suivant la profondeur de champ désirée (tableau 2).

On obtient donc un réseau de points donnant une image en fausses couleurs chacune correspondant à l'altitude relative de chaque point d'analyse de la surface (fig. 13).

\begin{tabular}{|l|c|c|}
\hline Profondeur de champ & $3000 \mu \mathrm{m}$ & $300 \mu \mathrm{m}$ \\
\hline Résolution en Z & $0,1 \mu \mathrm{m}$ & $0,01 \mu \mathrm{m}$ \\
\hline Résolution en X, Y & $0,1 \mu \mathrm{m}$ & $0,1 \mu \mathrm{m}$ \\
\hline
\end{tabular}

Tableau 2 : caractéristiques de résolution spatiale en fonction de l'optique utilisée

Table 1: Characteristis of spatial resolution for different opticals.

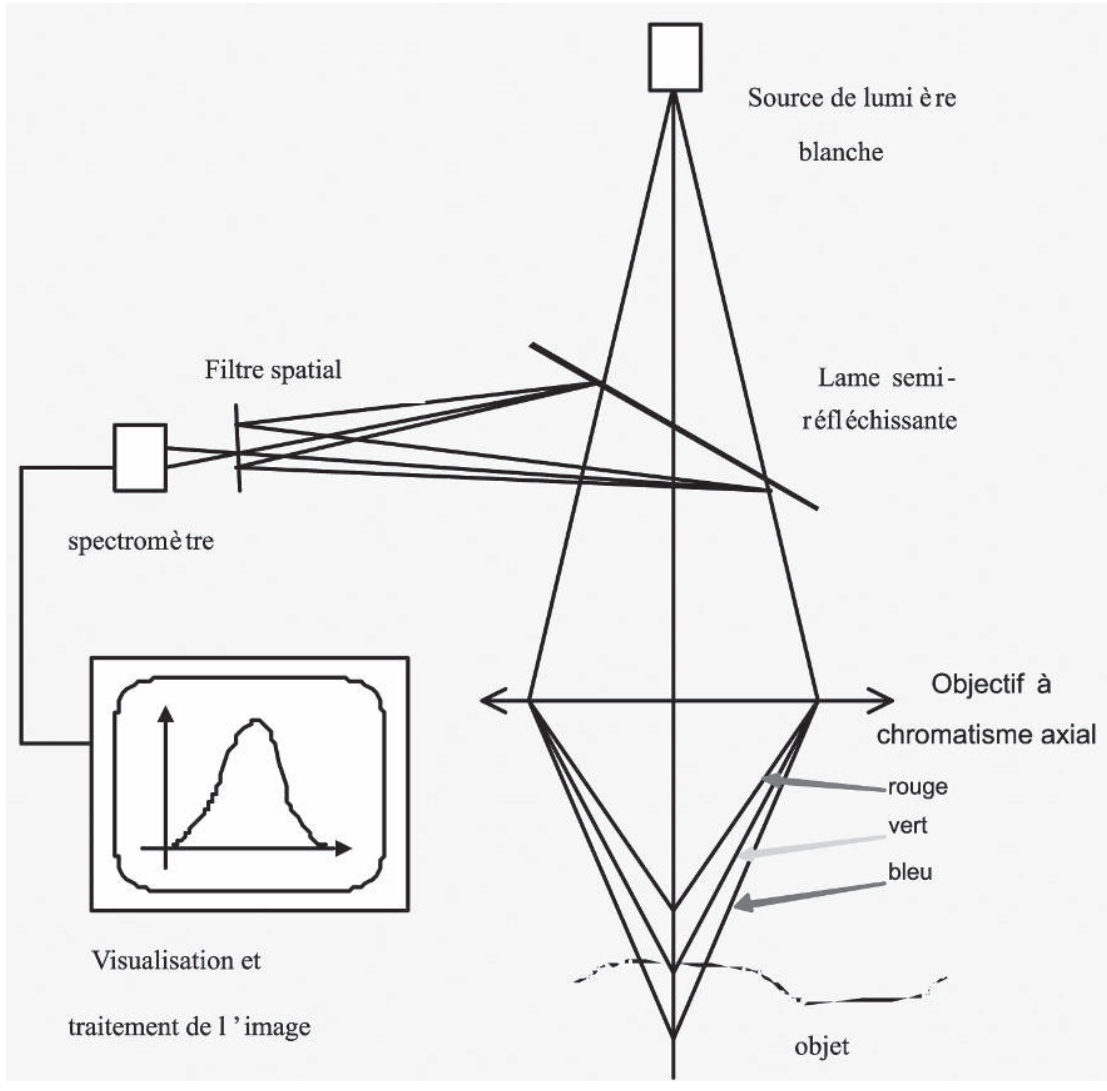

Figure 13 : Schéma de principe du microrugosimètre.

Figure 13: Scheme of the principle of the microrugosimeter. 


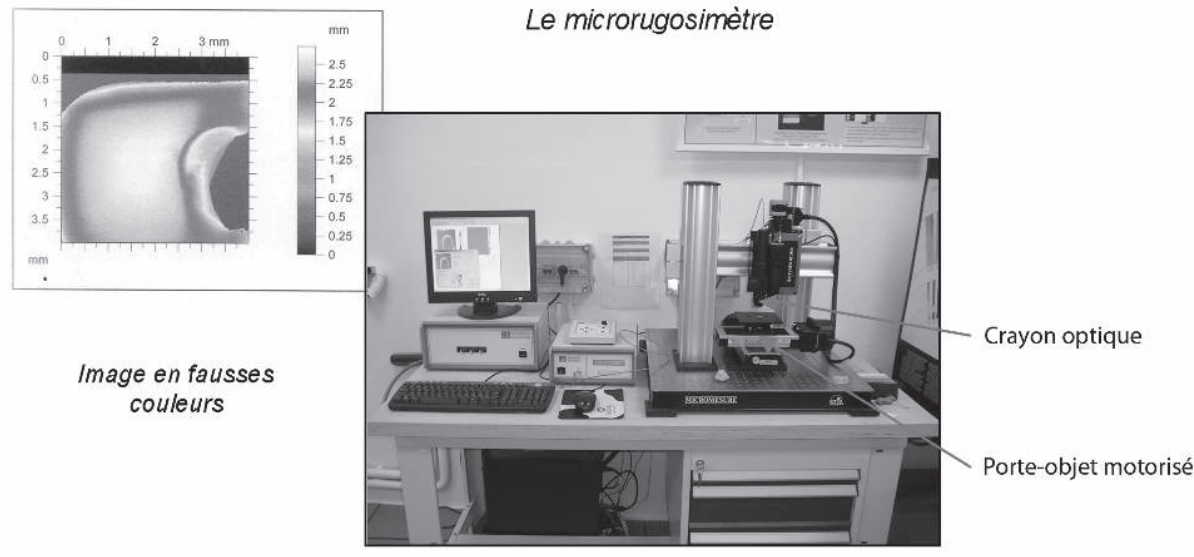

Figure 14 : (Voir planche couleur) Microrugosimètre STIL du C2RMF. Figure 14: (See colour plate) Microrugosimeter STIL of C2RMF.

\section{RÉSUltats ET DISCUSSION}

Nous avons pris pour exemple l'observation de la perle 30 de l'abri Pataud. De cette acquisition de mesures, un logiciel permet d'extraire des lignes de profil dans toutes les directions, il permet également d'afficher la valeur du rayon de courbure de la zone bombée, de même que l'angle d'ouverture de la perforation (fig. 15).

Les perles de l'abri Pataud ont des similitudes de dimensions mais diffèrent légèrement par leur forme (fig. 16) (Brickeer, 1995). Les unes sont bombées sur les deux faces, les autres ont un bombement sur une seule face, l'autre face est plane et légèrement arquée. Cette surface aurait pu être rabotée à partir de la forme précédente et donc amincir son épaisseur. Dans les deux cas la perforation est réalisée par semi-rotation de $-120^{\circ}$. Les angles d'ouverture de la perforation varient de $50^{\circ}$ à $75^{\circ}$ suivant les perles. Toutes n'ont pu être mesurées à cause de la présence de vernis protecteur qui masque les traces superficielles et agglomère les sédiments où se trouvaient ces perles.

Il y a analogie de forme avec des perles de la même époque Gravettienne : celles des Peyrugues (Allard et al., 1967) et celle du Blot (fig. 17). Toutes les trois présentent bulbes, ensellement et perforation biconique par semi-rotation, seules les dimensions different allant de $-7,5 \mathrm{~mm}$ pour les perles de l'abri Pataud, $10 \mathrm{~mm}$ pour celles des Peyrugues, à $13 \mathrm{~mm}$ pour celle du Blot. Pour certaines perles de l'abri Pataud seule une des faces est aplanie c'est le cas de la perle 24 (fig. 17), elles ont alors une plus faible épaisseur.

Il y a similitude de forme des perles de ces trois sites avec celles retrouvées au voisinage de sépultures à Sungir en Russie et à Grub/Krawetberg en Autriche (Antl, 2005). Elles sont datées de la même période et réalisées en os ou ivoire de mammouth (White, 1993) comme celles de l'abri Pataud des Peyrugues et du Blot.

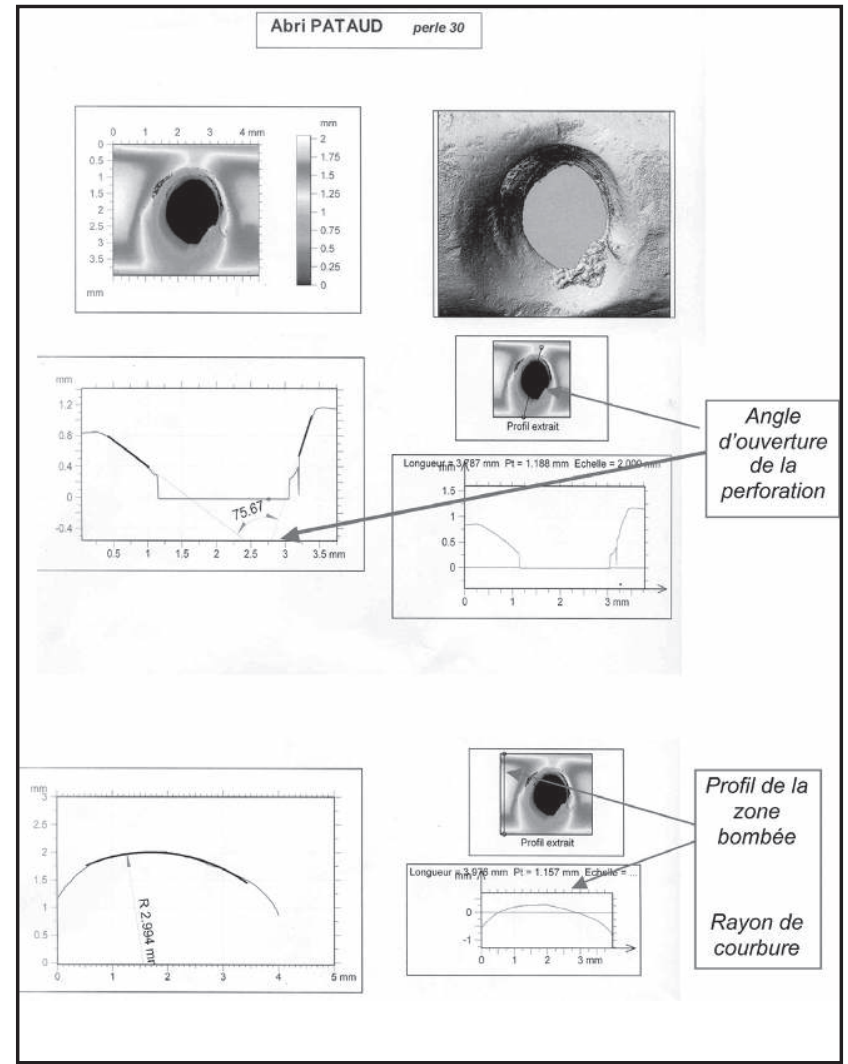

Figure 15: (Voir planche couleur) Perle 30 de l'abri Pataud: exemple de mesure de l'angle de perforation et du rayon de courbure du bombement de la perle.

Figure 15: (See colour plate) Bead n'30 of abri Pataud: measurement example of the perforation angle and of the radius of the convexity of the bead.

\section{Conclusions}

Avec ces équipements, complétés par l'expérimentation, il est possible de remonter au façonnage des perles préhistoriques et de tout objet perforé : 


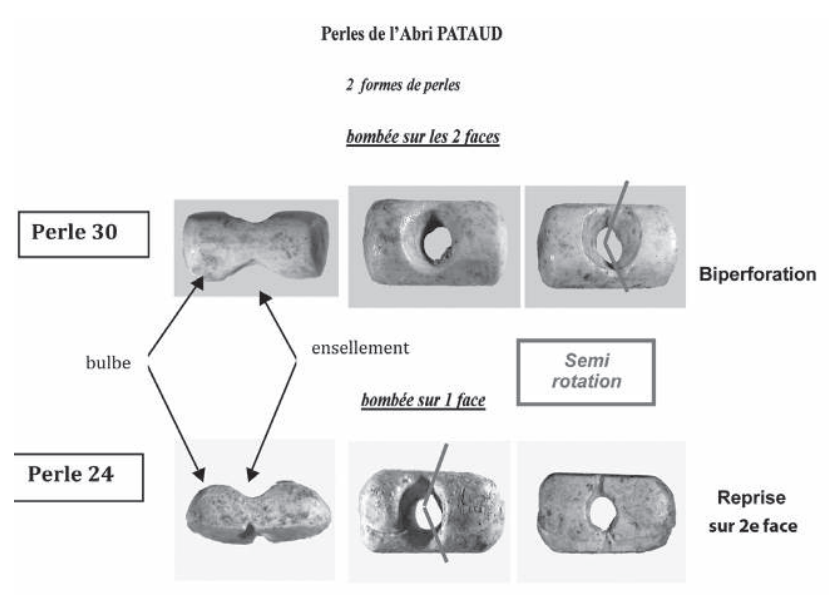

Figure 16 : (Voir planche couleur) Perles de l'abri Pataud. Figure 16: (See colour plate) Beads of the abri Pataud.

- à l'ouverture de la perforation,

- à l'angle d'incidence du perçoir par rapport à la surface,

- à l'angle de semi-rotation, donc à la tenue de l'outil pour réaliser ces perforations,

- aux rayons de courbure des surfaces bombées,

- aux stries et irrégularités de surface.

En cela Microscope Electronique à Balayage (MEB) et microrugosimètre sont très complémentaires. Le MEB a une grande profondeur de champ et peut donner la nature des éléments de surface de l'objet observé, le microrugosimètre permet de chiffrer les formes et les irrégularités de surface sans contact donc sans risque de dégradation.

Les hypothèses faites sur les paramètres liés à la perforation sont valables et l'expérimentation simule bien les perforations observées sur les perles archéologiques.

\section{BibliograPHIE}

Allard M., Drieux M., Jarry M., Pomies M.-P., Rodière J., 1997 - Perles en bois de renne du niveau 18 des Peyrugues..., Paléo, n 9, décembre 1997.

ANTL W., 2005 - L'industrie en os et en ivoire du site Gravettien à Grub/Kranawetberg près de Stillfried (note préliminaire), in Comportement des hommes du Paléolithique moyen et supérieur en Europe, D. Vialou et al. Acte du colloque du GDR 1945 du CNRS Paris 8-10 janvier 2003. Liège ERAUL 111, p. 51-58.

Brickeer H. M. (dir.), 1995 - Le Paléolithique supérieur de l'abri Pataud, DAF, Paris, Maison des Sciences de l'Homme.

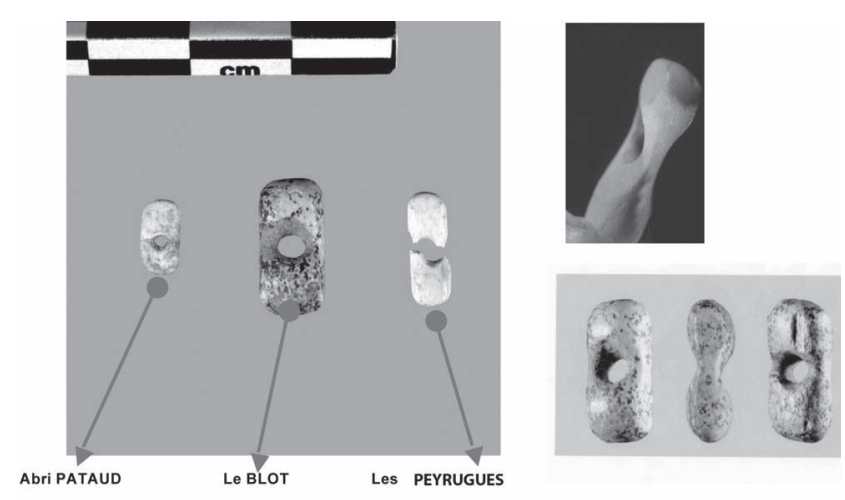

Figure 17 : (Voir planche couleur) Similitude de forme entre les perles des trois sites.

Figure 17: (See colour plate) Similarity of the form of the beads from the three sites.

Delporte H., 1972 - Proto-Magdalénien du Blot, commune de Cerzat (Haute Loire), Cong. Préhist. de Fr., XIX ${ }^{\mathrm{e}}$ sess., Auvergne, 1969 (1972). p. 190-199.

D'Errico, F., 1993 - Identification des traces de manipulation, suspension, polissage sur l'art mobilier en os, bois de cervidé, ivoire. Traces et fonction : les gestes retrouvés, Colloque international de Liège, Édition ERAUL.

MélarD, N., 2006 - Les pierres gravées du Magdalénien Moyen de La Marche (Lussac-les-Châteaux/Vienne) - Réalisation, fonctions et interprétations. Thèse de doctorat au Muséum National d'Histoire Naturelle, 2 vol., Paris.

Oтte M. 1981 - Le gravettien en Europe Centrale. vol. 1, De Temple Brugge.

RoDiÈre, J., 1996 - Façonnage de perles lithiques Magdaléniennes, Techne $n^{\circ} 3$, Ed LRMF/RMN.128, p. 54-62.

Stordeur, D., Pion, G., 1993 - La plaquette perforée en os de la Fru à Saint-Christophe (Savoie) : premier témoignage d'un forage alternatif, Gallia Préhistoire, tome 35 p. 293-304.

Taborin, Y., 2004 - Langage sans parole. La parure aux temps préhistoriques, Paris, La Maison des Roches, 215 p.

White, R., 1993 - Technological, and Social dimension of "Aurignacian-age". Body ornaments across Europe. In before Lascaux : the complex record of the early Upper Paleolothic, ed. H. Knecht, A. Pike-Tay, and R. White. Boca Raton, CRC Press, c, p. 277-299. 